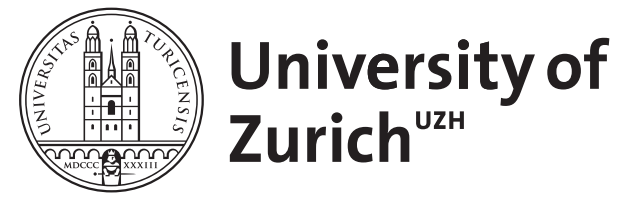

Zurich Open Repository and Archive

University of Zurich

University Library

Strickhofstrasse 39

CH-8057 Zurich

www.zora.uzh.ch

Year: 2013

Informatik ist auch eine Sozialwissenschaft!

Bernstein, Abraham

DOI: https://doi.org/10.1007/s00287-013-0722-z

Posted at the Zurich Open Repository and Archive, University of Zurich

ZORA URL: https://doi.org/10.5167/uzh-81729

Journal Article

Originally published at:

Bernstein, Abraham (2013). Informatik ist auch eine Sozialwissenschaft! Informatik-Spektrum, 36(5):461462.

DOI: https://doi.org/10.1007/s00287-013-0722-z 


\section{Informatik ist auch eine Sozialwissenschaft!}

\section{Abraham Bernstein}

Die Informatikbranche ist seit Jahren am Klagen: Es gäbe nicht genügend Studierende, zu wenig Spezialisten und die Bevölkerung interessiere sich nicht für unser Fach. Um diesen Missstand in den Griff zu bekommen, haben wir als Community schon verschiedenste Aktionen durchgeführt: das Jahr der Informatik in Deutschland, die Informatica08 in der Schweiz, Aktivitäten an Schulen, Greencards und vieles mehr.

So sass ich vor ein paar Wochen wieder einmal in einer illustren Runde, in der das Thema der Informatik an Schulen in der Schweiz diskutiert wurde. Dabei wurden Lehrpläne vorgestellt und Themen diskutiert, welche die Schüler in verschiedenen Altersklassen lernen sollten. Diese bestanden aus den uns allen bekannten Inhalten: Programmierung in verschiedensten Formen, Komplexität, Modellierung, und so weiter und so fort. Während ich mich nun von einem hervorragend zusammengesetzten und argumentativ ausgezeichnet konstruierten Lehrplan berieseln liess, vergegenwärtigte ich mir, wie die praktische Realität aussieht.

Hierbei verlasse ich mich allerdings nicht auf abgesicherte Informationen, sondern auf Introspektion - eine wissenschaftlich mit Vorsicht zu geniessende Vorgehensmethode - und zudem auf ein $N$ von zwei: meine eigene Schulerfahrung, die schon mehr als 25 Jahre zurück liegt, und diejenige meiner Tochter, die seit kurzem die erste Klasse des Gymnasiums besucht. Just vor einem halben Jahr hörte ich an einem Elternabend unter anderem, weshalb Latein keine tote Sprache sei. Die Lehrerin argumentierte wie folgt: Erstens sei Latein eine sehr lebendige Sprache, da sie ihr selbst das Leben ermögliche - das war der auflockernde Witz zur Einleitung. Doch dann wurde es ernst: Zweitens fördere Latein das abstrakte Denken. Es setze auf das Denken aufgrund einer Zusammensetzung von Fakten (auswendig gelernte Vokabeln) und Regeln (Konjugationsregeln, Grammatik, etc.) auf. Als ich dies hörte, dachte ich nur Informatik, Informatik und nochmals Informatik. Das Ganze war ja fast eine Zusammenfassung der ersten Kapitel einer Vorlesung zu wissensbasierten Systemen. Weshalb stürmen denn all diese Gymnasiastinnen und Gymnasiasten nicht in unsere Informatikstudiengänge?

Was mir bei all den Überlegungen vor allem bewusst wurde, ist, dass wir die Informatik aus Tradition immer wieder als Ingenieurwissenschaft oder als eine der Mathematik analogen Hilfswissenschaft definieren. Damit verlieren wir einen grossen Teil von möglichen Informatikstudierenden und schrecken einen noch grösseren Teil der Bevölkerung von unserer Wissenschaft $a b^{1}$. Einer Ende letzten Jahres veröffentlichten Studie der Pädagogischen Hochschule Zürich zufolge beschliessen Mädchen schon mit 14, dass sie kein Ingenieursstudium in Angriff nehmen wollen (Bieri et al.,

\footnotetext{
1 Salopp gesagt, wird immer wieder erwähnt: "Das iPhone kann ich ja auch ohne Studium bedienen." Und "Informatiker sind ja Ingenieure, die, im dunkeln Keller Pizza essend und Cola trinkend, möglichst ohne menschliche Kommunikation vor sich hin programmieren." Wir alle haben unsere eigene Sammlung abschreckender stereotypischer Anekdoten, die diese Vorurteile bekräftigen und in die eine oder andere Richtung weiterentwickeln.
} 
$2012^{2}$ ). Sie entscheiden sich zu diesem Zeitpunkt meist für ein Studium in den Sozialwissenschaften. Oberflächlich gesehen könnte man nun fragen, wie diese Affinität zu den Sozialwissenschaften abzuschwächen sei. Diese Frage greift aber meiner Meinung nach nicht tief genug. Sie aberkennt der Informatik eine ihr heute inhärente Dimension: die sozialwissenschaftliche.

Auch mir liegt die Ingenieursinformatik am Herzen. Der systematische Einsatz von mehreren Abstraktionsebenen, die fundierte Entwicklung von Prozessen auf solchen Abstraktionsebenen oder die methodische Modularisierung von Problemen zu Divide-and-Conquer Lösungen. All dies sind Konzepte, welche die traditionelle Informatik als Wissenschaft der Gesellschaft gegeben hat und die so viele Bereiche unseres Lebens nachhaltig beeinflusst haben. Was diese Sichtweise aber ignoriert, ist, dass der Computer schon längst ein soziales Artefakt geworden ist - eines der Hauptargumente aus Shoshana Zuboffs Buch "In the Age of the Smart Machine" aus dem Jahr 1989. Er hat Einfluss auf unsere soziale Umwelt, vermittelt und formt soziale Kontakte, gestaltet unsere Arbeitswelt und Freizeit, legitimiert unser Handeln und bestimmt Bedeutung sowie Wichtigkeit verschiedener Handlungen. Gleichzeitig wird ein Grossteil unserer Studienabgänger nicht Ingenieur, sondern Kommunikationsarbeiter: sie sprechen mit zukünftigen Nutzern und Auftraggebern, unterhalten sich über mögliche Anforderungen, sie gestalten soziale, durch Computer moderierte Interaktionen, ja sie erdenken sich Methoden, um menschliche Arbeit zu koordinieren - alles Tätigkeiten, die äusserst kommunikativ sind und einen sehr starken sozialwissenschaftlichen Charakter haben.

Doch es geht noch weiter. Bei uns in der Fakultät - die Informatik ist aus historischen Gründen ein Teil der wirtschaftswissenschaftlichen Fakultät der Universität Zürich - hat unlängst die Nachfrage nach informatischem Denken stark zugenommen. Die Methoden der rechnergestützten Sozialwissenschaften (englisch: Computational Social Science; siehe auch Lazer et al., 2009²) benötigen immer mehr Wirtschaftswissenschaftler, die sich in Informatik auskennen. Zudem sind immer mehr Wirtschaftsstudierende an Informatik interessiert. Die sozialwissenschaftlichen Aspekte ziehen also Personen in die Informatik, die sie vorher nicht mal mit einer Zange berührt hätten.

Weshalb halten wir uns denn krampfhaft an das Bild der Informatik, mit dem wir aufgewachsen sind? Sollten wir uns nicht gegenüber neuen Perspektiven unseres Feldes öffnen und uns wandeln? Insbesondere angesichts der Möglichkeiten, die sich für uns bieten (z.B. bei der Verbreiterung des Studierendenpools, der Themenvielfalt und des Impacts unserer Arbeiten) verstehe ich nicht, was dagegen spricht. Deshalb plädiere ich dafür, dass wir die sozialwissenschaftliche Dimension unserer Disziplin endlich vollumfänglich akzeptieren und als einen der Grundbausteine in unsere Ausbildungscurricula aufnehmen. Dabei müssten dann Aspekte wie zum Beispiel Mensch-Maschine-Interaktion, Social Computing oder sozialwissenschaftliche Methoden wie das Experimentieren mit Probanden und Umfragen feste Bestandteile unseres Pflichtprogramms werden, und bei der Einführung der Programmierung müsste eine Neuorientierung stattfinden - mit

\footnotetext{
2 Bieri Buschor, Christine; Berweger, Simone; Keck Frei, Andrea \& Kappler, Christa (2012). "Geschlechts(un-)typische" Studienwahl: Weshalb Frauen Ingenieurwissenschaften studieren und Männer Primarlehrer werden. Projektbericht. Pädagogische Hochschule Zürich.

3 David Lazer, Alex Pentland, Lada Adamic, Sinan Aral, Albert-László Barabási, Devon Brewer, Nicholas Christakis, Noshir Contractor, James Fowler, Myron Gutmann, Tony Jebara, Gary King, Michael Macy, DebRoy, and Marshall Van Alstyne. Computational Social Science, Science 6 February 2009: 323 (5915), 721 723. [DOI:10.1126/science.1167742]
} 
Schwerpunkten auf Gestaltung oder Erzählen, wie es an mehreren US-Hochschulen schon praktiziert wird.

Längerfristig hilft so ein Vorgehen bestimmt, das Bild unserer Disziplin zu wandeln und sie damit neuen Interessentinnen und Interessenten gegenüber zu öffnen, so dass die Informatik den ihr zustehenden Stellenwert in unserer Gesellschaft auch tatsächlich einnehmen kann.

\section{Danksagung}

Ich möchte mich herzlich bei Friedemann Mattern bedanken, der mich dazu ermutigt hat, meine Gedanken aufzuschreiben, und mir auch Feedback zum Text gab. 